\title{
Treatment of Mediastinitis due to Methicillin-resistant Staphylococcus aureus in a Renal Dysfunction Patient Undergoing Adjustments to the Linezolid Dose
}

\author{
Yasuhiro Tsuji ${ }^{1}$, Masato Tashiro ${ }^{2}$, Nobuyuki Ashizawa ${ }^{2}$, Yukio Ota ${ }^{1}$, Hayato Obi ${ }^{3}$, \\ Saori Nagura ${ }^{3}$, Munetoshi Narukawa ${ }^{2}$, Kazuaki Fukahara ${ }^{3}$, Naoki Yoshimura ${ }^{3}$, \\ Hideto $\mathrm{To}^{1}$ and Yoshihiro Yamamoto ${ }^{2}$
}

\begin{abstract}
This study is the first case report of the treatment of methicillin-resistant Staphylococcus aureus (MRSA) mediastinitis using therapeutic drug monitoring of the serum and wound exudate concentrations of linezolid in a renal dysfunction patient. In the present study, the serum trough concentration of linezolid was maintained between 2 and $7 \mu \mathrm{g} / \mathrm{mL}$. Therapeutic drug monitoring dosage adjustments may be especially useful in patients with renal dysfunction and severe MRSA infection.
\end{abstract}

Key words: linezolid, mediastinitis, methicillin-resistant Staphylococcus aureus, renal dysfunction, therapeutic drug monitoring

(Intern Med 54: 235-239, 2015)

(DOI: 10.2169/internalmedicine.54.2292)

\section{Introduction}

Although mediastinal infection rarely occurs after open heart surgery, such infections are associated with high morbidity and mortality as well as longer hospital stays (1). The most common etiological agents of mediastinal infection are Staphylococcus aureus and Staphylococcus epidermidis (2). Linezolid is a novel synthetic oxazolidinone antimicrobial agent with a different mechanism of action from that of other existing drugs that has shown to be effective for treating infections caused by multidrug-resistant Gram-positive cocci (3).

Adjustments to the dose of linezolid based on age, gender and a reduced renal function have previously been suggested to be unnecessary $(4,5)$. However, several studies have reported a correlation between thrombocytopenia and linezolid overexposure in patients with renal dysfunction (6-9). Furthermore, the prolonged use of linezolid has been shown to induce adverse hematological events $(10,11)$. Therefore, optimizing the dose of linezolid may be beneficial for preventing hematotoxicity and/or dose-dependent toxicity. Nevertheless, no clinical trials have yet evaluated the successful treatment of methicillin-resistant Staphylococcus aureus (MRSA) mediastinitis infections while simultaneously confirming the concentration of linezolid.

The aim of the present study was to treat mediastinitis using therapeutic drug monitoring of the serum and wound exudate concentrations of linezolid in a renal dysfunction patient. We herein describe the case of a cardiovascular surgery patient treated with linezolid who exhibited linezolidinduced thrombocytopenia.

\section{Case Report}

The patient was a 78-year-old Japanese man who weighed $48.2 \mathrm{~kg}$ and had a one pack-per-day smoking history for 42 years. He also had a history of diabetes and diabetic nephro-

\footnotetext{
${ }^{1}$ Department of Medical Pharmaceutics, Graduate School of Medicine and Pharmaceutical Sciences for Research, University of Toyama, Japan, ${ }^{2}$ Department of Clinical Infectious Diseases, Graduate School of Medicine and Pharmaceutical Sciences for Research, University of Toyama, Japan and ${ }^{3}$ Department of Surgery, Graduate School of Medicine, University of Toyama, Japan

Received for publication December 10, 2013; Accepted for publication June 29, 2014

Correspondence to Dr. Yoshihiro Yamamoto, yamamoto@med.u-toyama.ac.jp
} 

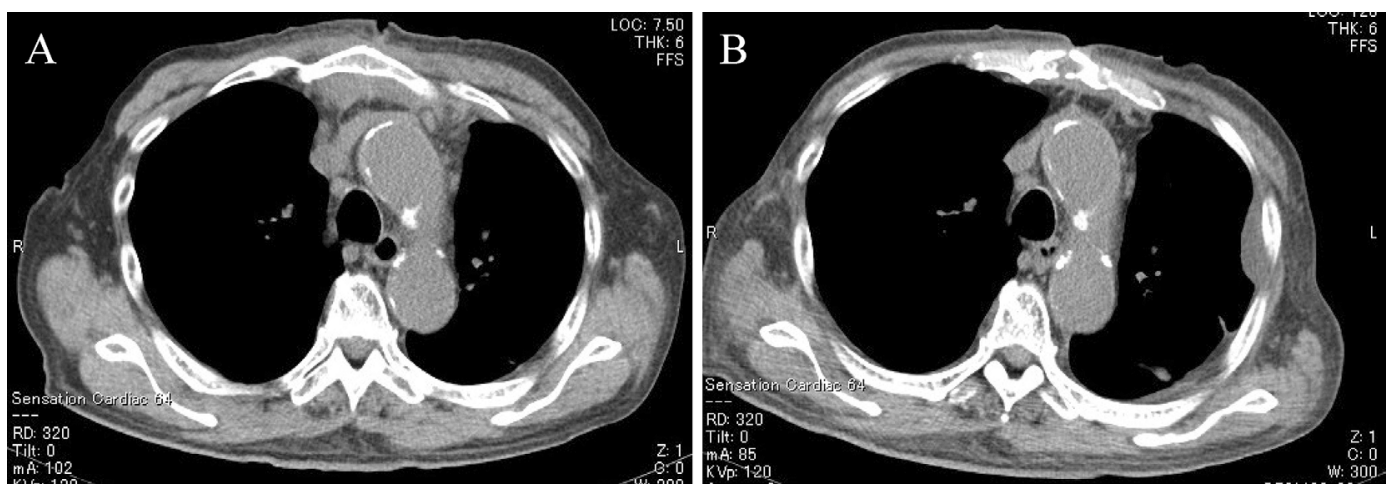

Figure 1. Chest computed tomography (CT). A. Chest CT performed immediately prior to the administration of linezolid. B. CT performed on the 55th day of linezolid tharepy

pathy, but dyslipidemia. He was admitted to our hospital for congestive heart failure and underwent coronary angiography. Consequently, severe stenosis was observed in all three branches of the coronary artery (\#2: 99\%, \#4PD: 75\%, \#6: 75-90\%, \#7: 75\%, \#9: 90\%) and the patient underwent coronary artery bypass grafting (CABG). We performed 3CABG (left internal thoracic artery-left anterior descending branch, aorta-saphenous vein-obtuse marginal branch, aortasaphenous vein-right coronary artery), a surgical technique used for off-pump coronary artery bypass. The operative time was approximately 3 hours and 50 minutes. Sulbactam/ ampicillin was administered to prevent perioperative infection.

Seven days after surgery, the patient's white blood cell count (WBC) increased to $9,770 / \mu \mathrm{L}$, the C-reactive protein (CRP) level increased to $7.0 \mathrm{mg} / \mathrm{dL}$ and he developed a fever (body temperature: $37.8^{\circ} \mathrm{C}$ ) and shaking chills. Treatment with levofloxacin was initiated on day 9 in the presence of pyuria, and the patient's fever was markedly ameliorated. On day 14, the sutures at the wounded site were removed, and partial protrusion of the central portion was detected. On day 16, pus exuded from this area when incised; however, cultures were negative. The abscess was subsequently opened using continuous debridement, as the flow of pus persisted. Three weeks after surgery, chest computed tomography (CT) revealed faint shadows in the anterior mediastinum on median sternotomy (Fig. 1A). Sternal osteomyelitis was not detected on CT; therefore, we diagnosed the patient as being without sternal osteomyelitis. In contrast, two sets of blood and pus cultures were positive for MRSA; thus, mediastinitis was diagnosed as a postoperative complication. The results of a laboratory examination were as follows: $\mathrm{WBC}=14,500 / \mu \mathrm{L}$ and $\mathrm{CRP}=14.5 \mathrm{mg} / \mathrm{dL}$. Wound cleaning, vacuum-associated closure (VAC) dressing insertion and linezolid administration as an antimicrobial agent were performed to control the purulent sternal MRSA infection and MRSA mediastinitis. This patient continued VAC for 30 days, without further thoracotomy incisions. Although he had end-stage renal disease, he had not received hemodialysis because he was able to spontaneously urinate regularly during the perioperative period. His blood urea nitro- gen level was $68 \mathrm{mg} / \mathrm{dL}$, his serum creatinine level was 5.6 $\mathrm{mg} / \mathrm{dL}$ and his glomerular filtration rate was $8.6 \mathrm{~mL} / \mathrm{min} /$ $1.73 \mathrm{~m}^{2}$, which was estimated using an equation corrected with a coefficient for Japanese patients. Therefore, linezolid was administered at a dose of $600 \mathrm{mg}$ via intravenous drip infusion for 60 minutes at 24-hour, instead of 12-hour, intervals. Blood and wound exudates were collected immediately prior to the administration of linezolid and 24 hours later in order to measure the trough concentration $\left(\mathrm{C}_{\min }\right)$. After the initiation of linezolid treatment, the serum $\mathrm{C}_{\min }$ was assumed to be maintained between 2 and $7 \mu \mathrm{g} / \mathrm{mL}(11,12)$. The dose of linezolid administered was carefully adjusted based on the linezolid $\mathrm{C}_{\min }$.

The measurement of the serum and wound exudate linezolid concentrations using high-performance liquid chromatography (HPLC) has been previously described (13). The bulk powder of linezolid for HPLC was provided by Pfizer (Peapack, USA), and the lower limit for the detection of linezolid using this method was $0.1 \mu \mathrm{g} / \mathrm{mL}$ with intra/interday precision below $5.0 \%$. The minimum inhibitory concentration (MIC) of isolated MRSA was determined using the E-test, which was performed according to the manufacturer's instructions. The linezolid MIC of the MRSA isolates was $1 \mu \mathrm{g} / \mathrm{mL}$. The relationships between the platelet count, serum and wound exudate $\mathrm{C}_{\min }$ values and adjustments to the dose of linezolid are shown in Fig. 2. The platelet count was $20.5 \times 10^{4} / \mu \mathrm{L}$ on the first day of linezolid therapy. On the 21 st day of therapy, the serum $C_{\min }$ increased to $11.5 \mu \mathrm{g} / \mathrm{mL}$, while the platelet count decreased to $6.5 \times 10^{4} / \mu \mathrm{L}$, at which time the patient developed severe thrombocytopenia. Furthermore, the platelet count decreased as the serum $\mathrm{C}_{\min }$ increased. Platelet clumping was not observed on Giemsa staining, and there was no evidence of disseminated intravascular coagulopathy or idiopathic thrombocytopenic purpura. The final diagnosis was linezolid-induced thrombocytopenia. The cessation of linezolid treatment for one day led to a reduction in the serum $\mathrm{C}_{\min }$ to $3.5 \mu \mathrm{g} / \mathrm{mL}$ on the 23 rd day of linezolid therapy (platelet count: $6.4 \times 10^{4} / \mu \mathrm{L}$ ). Therefore, the linezolid treatment was resumed at a dose of $600 \mathrm{mg}$ daily; however, the serum $\mathrm{C}_{\min }$ increased to $9.3 \mu \mathrm{g} /$ $\mathrm{mL}$ on the $27 \mathrm{th}$ day of linezolid therapy (platelet count: 


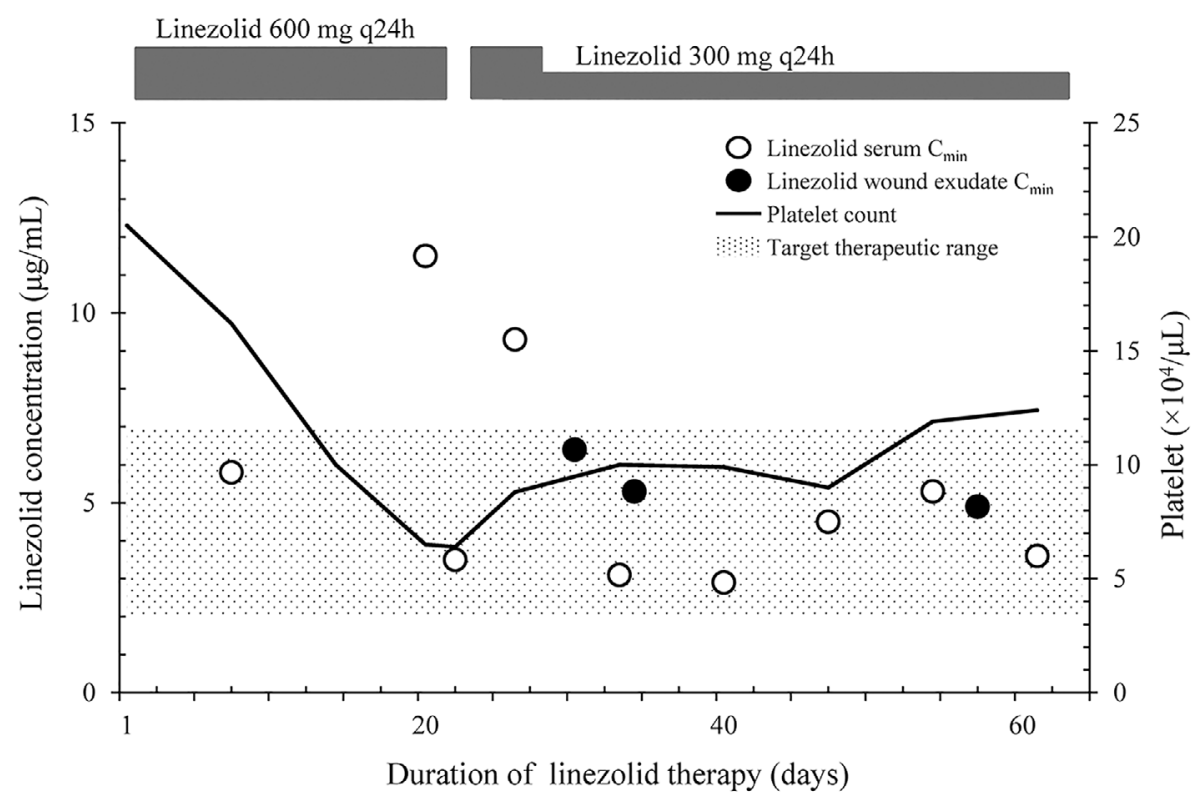

Figure 2. Relationship between the platelet counts and linezolid trough concentrations in the serum and wound exudate. q24h: every 24 hours

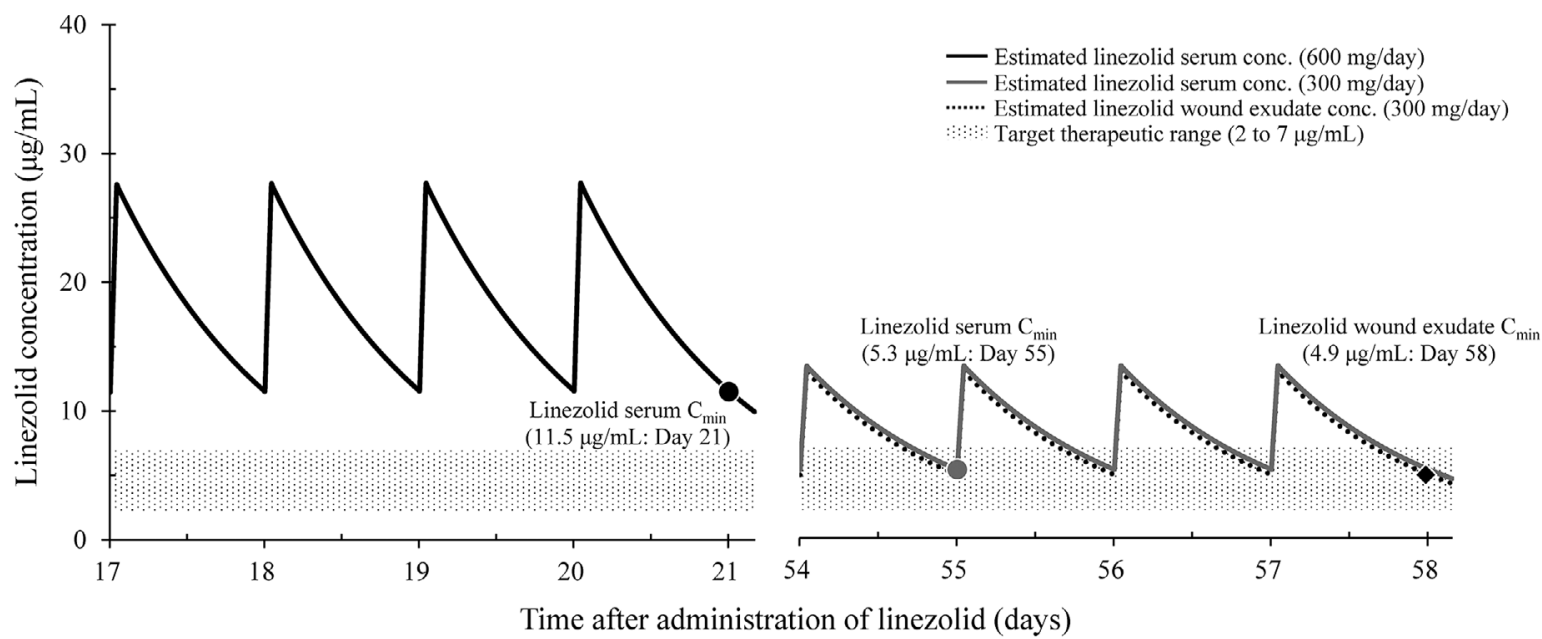

Figure 3. Concentration-time profiles and simulation curves of the linezolid concentrations in the serum and wound exudate

$\left.8.8 \times 10^{4} / \mu \mathrm{L}\right)$. We thus decreased the dose of linezolid to 300 $\mathrm{mg}$ (the thrombocytopenia was not exacerbated when the serum $\mathrm{C}_{\min }$ was between 2 and $7 \mu \mathrm{g} / \mathrm{mL}$ in the target therapeutic range), and the platelet count gradually recoverd.

The estimated linezolid concentrations in the serum on the $21 \mathrm{st}$ ( $600 \mathrm{mg} /$ day) and 55th days of linezolid therapy (300 mg/day) and the wound exudate on the 58th day of linezolid therapy (300 mg/day), as simulated according to the Bayesian non-linear least squares method, and the observed linezolid concentrations are shown in Fig. 3. The serum $\mathrm{C}_{\min }$, concentration-time profiles and simulation curves measured at the trough level on the 55th day of linezolid therapy were similar to those for the wound exudate on the 58th day of linezolid therapy.

Pus cultures were negative on the 35th and 54th days of linezolid therapy. The size of the wound markedly decreased by the 60th day of linezolid therapy, with no redness observed along the periphery. The granulation in the ulcer floor was favorable, although exudate was continuously observed, as complete epithelization had not yet been obtained. On the other hand, CT revealed a clear reduction in the size of the soft tissue shadow in the mediastinal region (Fig. 1B). No fever was detected, and the CRP level was $0.04 \mathrm{mg} / \mathrm{dL}$ on the 64th day of linezolid therapy, which indicated remission of the patient's inflammatory reaction. No further worsening of the platelet count was noted (platelet count: $12.4 \times 10^{4} / \mu \mathrm{L}$ ). Therefore, we concluded that the inflammation caused by the purulent sternal MRSA infection and MRSA mediastinitis had been resolved by the linezolid treatment, and the drug was discontinued. Consequently, 
there were no additional episodes of MRSA mediastinitis, and the patient was discharged at the end of linezolid administration on day 21 with a favorable prognosis.

\section{Discussion}

The onset of mediastinitis following cardiovascular surgery remains an intractable complication associated with considerable mortality. Dodds et al. reported (14) that a female gender, elderly status ( $>75$ years of age) and diabetes are risk factors for the development of postoperative mediastinitis due to MRSA. In addition, an older age and diabetes were suspected risk factors for mediastinitis in our patient. The degree of penetration of antibiotic agents, such as vancomycin, into the mediastinal space is known to be low because this region is separated from the bloodstream. However, Sacar et al. showed that both vancomycin and linezolid successfully reduced the bacterial count in the mediastinum and at the sternotomy line in rats (15) and that linezolid is easily transferred from the blood to the mediastinum and breast tissue in human patients (16). Therefore, we gave preference to treatment with linezolid in the present case. In this study, mediastinitis due to MRSA in a renal dysfunction patient was treated using therapeutic drug monitoring of the linezolid levels based on the serum and wound exudate $\mathrm{C}_{\min }$ values.

Linezolid-induced adverse events, such as thrombocytopenia, resulting from persistently high linezolid concentrations have been described in patients with renal dysfunction $(8,9,11)$. These studies reported that the linezolid $\mathrm{C}_{\min }$ values are significantly higher in patients with renal impairment than in those without. For example, Nukui et al. noted that thrombocytopenia developed more frequently in patients with a linezolid trough concentration of $>7.5 \mu \mathrm{g} / \mathrm{mL}$ (11). In the present case, despite decreasing the daily dose of linezolid from 1,200 to $600 \mathrm{mg}$ at the start of treatment to prevent thrombocytopenia, the $\mathrm{C}_{\min }$ of linezolid in the serum was $11.5 \mu \mathrm{g} / \mathrm{mL}$ on $21 \mathrm{st}$ day of linezolid therapy, which was sufficient to induce thrombocytopenia. This finding demonstrates the importance of dose adjustment in patients with severe renal failure. Therapeutic drug monitoring is therefore important for enabling adjustments of the drug dose for better safety and efficacy. A recent small retrospective study reported the beneficial effects of therapeutic drug monitoring with respect to the safety outcomes of patients undergoing long-term treatment with linezolid (12). The authors showed that thrombocytopenia can be resolved by adjusting the dose of linezolid based on the results of therapeutic drug monitoring and suggested that maintaining the serum $\mathrm{C}_{\min }$ value between 2 and $7 \mu \mathrm{g} / \mathrm{mL}$ improves safety outcomes while retaining appropriate efficacy. However, the results of the present and previous studies were limited to only a few samples (12). Therefore, further research of the efficacy of therapeutic drug monitoring of linezolid is warranted.

We performed therapeutic drug monitoring of the $C_{\min }$ in the serum based on the above findings and found that this strategy prevented the exacerbation of thrombocytopenia in our patient, with little or no loss in efficacy due to dose reduction. Furthermore, the $\mathrm{C}_{\min }$ of linezolid was similar between the wound exudate and serum. Previous studies investigating the pharmacokinetics of linezolid in patients have shown that changes in the renal function significantly affect the pharmacokinetics of linezolid $(7,17)$. The present patient's glomerular filtration rate increased from 5.7 to 11.5 $\mathrm{mL} / \mathrm{min} / 1.73 \mathrm{~m}^{2}$ during the administration of linezolid, which may have induced the small variations observed in the $\mathrm{C}_{\min }$ of linezolid after the daily dose of linezolid was changed from 600 to $300 \mathrm{mg} /$ day.

A low $\mathrm{C}_{\min }$ of linezolid in the serum may result in treatment failure. However, the individual serum and wound exudate $\mathrm{C}_{\min }$ values of linezolid in the present case were higher than the MIC of $1 \mu \mathrm{g} / \mathrm{mL}$ at all measurement timepoints. These results are consistent with the findings of a small number of previous studies of adult and pediatric patients in which linezolid was shown to be effective against MRSA mediastinitis $(16,18)$.

Cossu et al. recently reported that further studies should be conducted in order to determine whether the incidence of linezolid-related thrombocytopenia can be reduced using dose adjustments based on the renal function (19). We performed the current study to establish whether it is necessary to consider dose adjustments, and the results subsequently confirmed that adjustments based on therapeutic drug monitoring successfully cured the patient's infection.

In conclusion, we herein demonstrated that linezolid therapeutic drug monitoring in a patient with renal failure enabled the continuation of linezolid therapy, suggesting the possible beneficial effects in ameliorating thrombocytopia using dose adjustments of linezolid. Despite the reduced concentration of linezolid observed in the patient's serum, the concentration in the wound exudate was sufficient to inhibit the growth of MRSA. It is beneficial for clinicians to understand the effects of therapeutic drug monitoring of linezolid in renal dysfunction patients in cases in which no alternative drugs to linezolid can be used to continuously treat MRSA. However, it can be difficult to measure the linezolid concentration. Therefore, it is necessary to develop simple and easy methods for measuring the blood concentration of linezolid in community hospitals. The results of the present study provide new insight into the appropriate dose and administration schedule of linezolid in renal dysfunction patients.

This study was performed in accordance with the Declaration of Helsinki after receiving approval by the Ethics Committee of the University of Toyama. The study protocol was explained to the patient in writing, who then provided his written informed consent. The patient's privacy was fully protected, and his personal information was handled such that he could not be identified.

Author's disclosure of potential Conflicts of Interest (COI). Yoshihiro Yamamoto: Honoraria, Pfizer. 


\section{References}

1. Farinas MC, Gald Peralta F, Bernal JM, Rabasa JM, Revuelta JM, Gonzalez-Macias J. Suppurative mediastinitis after open-heart surgery: a case-control study covering a seven-year period in Santander, Spain. Clin Infect Dis 20: 272-279, 1995.

2. Gardlund B, Bitkover CY, Vaage J. Postoperative mediastinitis in cardiac surgery: microbiology and pathogenesis. Eur J Cardiothorac Surg 21: 825-830, 2002.

3. Zurenko GE, Yagi BH, Schaadt RD, et al. In vitro activities of U100592 and U-100766, novel oxazolidinone antibacterial agents. Antimicrob Agents Chemother 40: 839-845, 1996.

4. Brier ME, Stalker DJ, Aronoff GR, et al. Pharmacokinetics of linezolid in subjects with renal dysfunction. Antimicrob Agents Chemother 47: 2775-2780, 2003.

5. Sisson TL, Jungbluth GL, Hopkins NK. Age and sex effects on the pharmacokinetics of linezolid. Eur J Clin Pharmacol 57: 793797, 2002.

6. Hiraki Y, Tsuji Y, Hiraike M, et al. Correlation between serum linezolid concentration and the development of thrombocytopenia. Scand J Infect Dis 44: 60-64, 2012.

7. Tsuji Y, Yukawa E, Hiraki Y, et al. Population pharmacokinetic analysis of linezolid in low body weight patients with renal dysfunction. J Clin Pharmacol 53: 967-973, 2013.

8. Wu VC, Wang YT, Wang CY, et al. High frequency of linezolidassociated thrombocytopenia and anemia among patients with endstage renal disease. Clin Infect Dis 42: 66-72, 2006.

9. Tsuji Y, Hiraki Y, Matsumoto K, et al. Thrombocytopenia and anemia caused by a persistent high linezolid concentration in patients with renal dysfunction. J Infect Chemother 17: 70-75, 2011.

10. Attassi K, Hershberger E, Alam R, Zervos MJ. Thrombocytopenia associated with linezolid therapy. Clin Infect Dis 34: 695-698, 2002.
11. Nukui $Y$, Hatakeyama S, Okamoto K, et al. High plasma linezolid concentration and impaired renal function affect development of linezolid-induced thrombocytopenia. J Antimicrob Chemother 68: 2128-2133, 2013.

12. Pea F, Viale P, Cojutti P, Del Pin B, Zamparini E, Furlanut M. Therapeutic drug monitoring may improve safety outcomes of long-term treatment with linezolid in adult patients. J Antimicrob Chemother 67: 2034-2042, 2012.

13. Majcher-Peszynska J, Haase G, Sass M, et al. Pharmacokinetics and penetration of linezolid into inflamed soft tissue in diabetic foot infections. Eur J Clin Pharmacol 64: 1093-1100, 2008.

14. Dodds Ashley ES, Carroll DN, Engemann JJ, et al. Risk factors for postoperative mediastinitis due to methicillin-resistant Staphylococcus aureus. Clin Infect Dis 38: 1555-1560, 2004.

15. Sacar S, Sacar M, Aybek H, et al. Comparison of the therapeutic efficacy of linezolid and vancomycin and correlation of serum and tissue malondialdehyde and myeloperoxidase in an experimental mediastinitis model. J Surg Res 152: 89-95, 2009.

16. Tsuji Y, Hashimoto W, Taniguchi S, et al. Pharmacokinetics of linezolid in the mediastinum and pleural space. Int $\mathrm{J}$ Infect Dis 17: e1060-e1061, 2013.

17. Sasaki T, Takane H, Ogawa K, et al. Population pharmacokinetic and pharmacodynamic analysis of linezolid and a hematologic side effect, thrombocytopenia, in Japanese patients. Antimicrob Agents Chemother 55: 1867-1873, 2011.

18. Kosaka T, Kokufu T, Shime N, et al. Pharmacokinetics and tolerance of linezolid for meticillin-resistant Staphylococcus aureus mediastinitis in paediatric patients. Int $\mathbf{J}$ Antimicrob Agents 33: 368-370, 2009.

19. Cossu AP, Musu M, Mura P, De Giudici LM, Finco G. Linezolidinduced thrombocytopenia in impaired renal function: is it time for a dose adjustment? A case report and review of literature. Eur J Clin Pharmacol 70: 23-28, 2014.

(C) 2015 The Japanese Society of Internal Medicine http://www.naika.or.jp/imonline/index.html 